\title{
Improved and Perfect Actions in Discrete Gravity
}

\author{
Benjamin Bahr ${ }^{1}$ and Bianca Dittrich ${ }^{2}$ \\ ${ }^{1}$ DAMTP, University of Cambridge, \\ Wilberforce Road, Cambridge CB3 0WA, UK \\ 2 MPI f. Gravitational Physics, Albert Einstein Institute, \\ Am Mühlenberg 1, D-14476 Potsdam, Germany \\ and \\ Institute for Theoretical Physics, Utrecht University, \\ Leuvenlaan 4, NL-3584 CE Utrecht, The Netherlands
}

November 4, 2018

\begin{abstract}
We consider the notion of improved and perfect actions within Regge calculus. These actions are constructed in such a way that they - although being defined on a triangulation - reproduce the continuum dynamics exactly, and therefore capture the gauge symmetries of General Relativity. We construct the perfect action in three dimensions with cosmological constant, and in four dimensions for one simplex. We conclude with a discussion about Regge Calculus with curved simplices, which arises naturally in this context.
\end{abstract}

\section{Introduction}

In General Relativity the notion of diffeomorphism invariance, resulting from Einstein's covariance principle, is of ultimate importance [1]. In particular, its correct implementation on the quantum level is a challenging task for every candidate quantum gravity theory.

Prior to quantizing a classical field theory, it is usually first discretized, since discrete systems generically have finitely many degrees of freedom. These are usually easier to quantize than the infinitely many degrees of freedom of field theories. A natural discretization of General Relativity is achieved by Regge calculus, where the smooth space-time is replaced by a simplicial complex, and the metric information is contained in the edge lengths and deficit angles around the hinges [2, 3]. A similar discretization is used within the Spin Foam quantization approach, where the variables of the first order Plebanski formulation of GR are discretized on Regge triangulations, prior to quantization [4].

It is an important question what happens with the diffeomorphism invariance of General Relativity in these discretized gravity theories (see [5] and references therein). 
Discretizing a theory often breaks symmetries, such as in QCD, where the introduction of a lattice breaks i.e. rotation invariance. Another example are reparametrization invariant one-dimensional systems, where the discretization scheme generically breaks the reparametrization invariance [6]. The latter example resembles the situation in GR in many ways [7].

In a canonical formulation the problem becomes even more apparent, where the symmetries turn into constraints, and it is notoriously difficult to implement them correctly in the discretized quantum theory (see also [8] for a discussion). Even in quantum gravity theories like LQG, which are inherently set out to capture the full continuum physics, the discretized nature of the constituents, i.e. the graphs, make the implementation of the constraint algebra rather non-trivial [5, 9].

In general, breaking of symmetries is, however, not ultimately tied to the discretization, but rather the approximation involved, i.e. by replacing spatial derivatives with differential quotients between neighboring lattice points.

For instance in lattice QCD one ideally would want to construct a Lagrangian which, although describing a theory on the lattice, still encodes the symmetries of the continuum theory [10, 11]. A lattice action which reproduces the same dynamics as the continuum theory and therefore also reflects the symmetries of the continuum limit is termed perfect action in that context. That perfect actions exist for asymptotically free theories follows from Wilson's theory of renormalization group flow [12. Although for actual problems at hand the perfect actions are very hard to compute, the (numerical) computation of improved actions, i.e. actions that capture the continuum symmetries much better than the actual naïve lattice discretization, is an important task. These actions are widely sought for in order to suppress lattice artifacts in numerical calculations [13].

In this article we investigate the question of how improved and perfect actions within the context of discretizations of General relativity, in particular Regge calculus, can be constructed. We will start with reviewing one-dimensional reparametrization invariant systems and their discretization in chapter 2. These systems exhibit a gauge symmetry which mimics diffeomorphism symmetry of GR in many respects. This symmetry is broken in the naïve discretization of those systems, and we will have a look at how one can construct improved and perfect actions for them. In particular we will see how the perfect actions restore the gauge invariance of the continuum limit within the discretized setting. Part of this chapter will follow [6].

In chapters 3 and 4.1 we will focus on Regge calculus with a cosmological constant in three and four dimensions. Whereas Regge calculus in $3 D$ with $\Lambda=0$ exhibits a wellknown vertex displacement symmetry which is a result of the discrete Bianchi identities [14, 15], this symmetry is broken for $\Lambda \neq 0$. We show how to construct improved actions in this case and analytically compute the perfect action, which regains the vertex displacement symmetry and hence reflects the dynamics and the symmetry of the continuum, albeit formulated on a Regge triangulation.

We also formulate improved actions for Regge calculus in $4 D$, and investigate some properties of its continuum limit, i.e. the corresponding perfect action. In particular we are able to show that the perfect action from the Regge action, and the one obtained by using simplices of constant curvature instead of internally flat ones, coincide. In the 
language of renormalization group flow this demonstrates that the two actions one started with lie in the same universality class.

We will in particular comment about the conclusions one can draw from these findings for the corresponding quantum theories.

\section{Discretized actions in 1D}

In this section we will discuss theories arising from discretizations of systems with onedimensional reparametrization invariance, that is invariance under redefinitions of the time variable. As we will see under discretization the exact reparametrization invariance is typically lost similar to the diffeomorphism invariance in the Regge action. However for the examples we consider in this section there is a procedure to obtain a discrete action with an exact reparametrization invariance. This procedure resembles the "blocking from the continuum" construction in lattice QCD, where a lattice action is constructed which has the exact symmetries of the continuum action [13]. In some parts of our discussion we will follow [6].

We start from a regular Lagrangian $L(q, \dot{q})$ where $q$ denotes the configuration variable. We assume that the dynamics determined by $L$ leads to a unique solution $q(t)$ for given boundary values $q\left(t_{i}\right), q\left(t_{f}\right)$ if $t_{i}$ and $t_{f}$ are sufficiently close together.

From this we construct a reparametrization invariant action by adding the time variable $t$ to the configuration variables and use $s$ as an (auxiliary) evolution parameter instead. If we define

$$
\tilde{L}\left(t, q, t^{\prime}, q^{\prime}\right):=L\left(q, \frac{q^{\prime}}{t^{\prime}}\right) t^{\prime},
$$

where a prime denotes differentiation with respect to $s$, then it is then straightforward to verify that

$$
S=\int_{s_{i}}^{s_{f}} \tilde{L}\left(t, q, t^{\prime}, q^{\prime}\right) d s
$$

is indeed invariant under reparametrizations $\tilde{s}=f(s)$ of the evolution parameter and the induced change $\tilde{t}(\tilde{s})=t\left(f^{-1}(\tilde{s})\right), \tilde{q}(\tilde{s})=q\left(f^{-1}(\tilde{s})\right)$ of the evolution paths.

The Euler-Lagrange equations for (2.2) for the variables $t, q$ are given by

$$
\begin{aligned}
& \left(\frac{\partial \tilde{L}}{\partial q}-\frac{d}{d s} \frac{\partial \tilde{L}}{\partial q^{\prime}}\right)=\frac{\partial L}{\partial q} t^{\prime}-\frac{d}{d s} \frac{\partial L}{\partial \dot{q}}=\left(\frac{\partial L}{\partial q}-\frac{d}{d t} \frac{\partial L}{\partial \dot{q}}\right) t^{\prime} \\
& \left(\frac{\partial \tilde{L}}{\partial t}-\frac{d}{d s} \frac{\partial \tilde{L}}{\partial t^{\prime}}\right)=-\frac{d}{d s} L+\frac{\partial L}{\partial q} q^{\prime}+\frac{\partial L}{\partial \dot{q}} \frac{d}{d s} \frac{d q^{\prime}}{d t^{\prime}}=\left(-\frac{d L}{d t}+\frac{\partial L}{\partial q} \dot{q}+\frac{\partial L}{\partial \dot{q}} \ddot{q}\right)(\mathbb{E} .4)
\end{aligned}
$$

where $\frac{\partial L}{\partial q}$ and $\frac{\partial L}{\partial \dot{q}}$ denote the derivative of $L$ w.r.t. its first and its second entry respectively. Note that (2.3) is equivalent to the Euler-Lagrange equations for $L$, and (2.4) is 
satisfied identically due to the chain rule. So this is just a reformulation of the dynamics determined by $L$ via introduction of a gauge degree of freedom. The non-uniqueness of the solutions $t(s), q(s)$ directly corresponds to the reparametrization independence of the dynamical system defined by the Lagrangian $\tilde{L}$.

A naïve discretization of the action (2.2) is given by

$$
S_{d}=\sum_{n=0}^{N-1}\left(t_{n+1}-t_{n}\right) L_{n}
$$

with

$$
L_{n}:=L\left(q_{n}, \frac{q_{n+1}-q_{n}}{t_{n+1}-t_{n}}\right) .
$$

The dynamics of this discretized system is obtained by looking for stationary variations of (2.5) w.r.t the $t_{n}, q_{n}$. The equations of motion are

$$
\begin{aligned}
0=\frac{\partial S_{d}}{\partial q_{n}}= & \partial_{q} L_{n}\left(t_{n+1}-t_{n}\right)+\partial_{\dot{q}} L_{n-1}-\partial_{\dot{q}} L_{n} \\
0=\frac{\partial S_{d}}{\partial t_{n}}= & L_{n-1}-L_{n}+\partial_{\dot{q}} L_{n} \frac{q_{n+1}-q_{n}}{t_{n+1}-t_{n}} \\
& -\partial_{\dot{q}} L_{n-1} \frac{q_{n}-q_{n-1}}{t_{n}-t_{n-1}}
\end{aligned}
$$

where $\partial_{q} L_{n}$ denotes the derivative $\frac{\partial L}{\partial q}$ evaluated at $q=q_{n}, \dot{q}_{n}=\frac{q_{n+1}-q_{n}}{t_{n+1}-t_{n}}$. Similarly $\partial_{\dot{q}} L_{n}$ is the derivative of $L$ w.r.t. its second entry evaluated at $\left(q_{n}, \dot{q}_{n}\right)$. With the product rule $A_{n+1} B_{n+1}-A_{n} b_{n}=A_{n+1}\left(B_{n+1}-B_{n}\right)+A_{n}\left(B_{n+1}-B_{n}\right)$, equation (2.8) for the $t_{n}$ can, using (2.7), be rewritten as

$$
\begin{aligned}
0 & =-\frac{L_{n}-L_{n-1}}{t_{n+1}-t_{n}}+\partial_{q} L_{n} \frac{q_{n+1}-q_{n}}{t_{n+1}-t_{n}} \\
& +\partial_{\dot{q}} L_{n-1} \frac{1}{t_{n+1}-t_{n}}\left(\frac{q_{n+1}-q_{n}}{t_{n+1}-t_{n}}-\frac{q_{n}-q_{n-1}}{t_{n}-t_{n-1}}\right) .
\end{aligned}
$$

In the continuum limit (2.9) converges to

$$
-\frac{d L}{d t}+\frac{\partial L}{\partial q} \dot{q}+\frac{\partial L}{\partial \dot{q}} \ddot{q}
$$

which vanishes identically, and is equivalent to (2.4). In the discrete case however, the equations of motion (2.9) for the $t_{n}$ do not vanish in general. So the equations for the $t_{n}$ are nontrivial, and have to be solved along with the $q_{n}$. Since the equations (2.9) only couple $t_{n}$ at most two steps apart from each other, the discrete system is of second order and generically imposing boundary values $t_{0}, q_{0}, t_{N}, q_{N}$ uniquely determines a solution. 
As a consequence, the discrete system defined by the action (2.5) does not capture the reparametrization invariance of the continuum dynamics defined by (2.2). One can show that this is directly linked to the failure of energy conservation within the the discrete system [6, 7, 8].

The loss of reparametrization invariance is, however, not ultimately tied to the discretization itself, but rather to the approximation (2.6). If, however, one can find a discrete action that exactly reproduces the continuum dynamics, one can regain the reparametrization freedom. Such actions are termed perfect actions e.g. in lattice gauge theory 1 . In the following chapter we will show how to construct a perfect action for the $1 D$ systems discussed above, in order to restore reparametrization invariance.

\subsection{Regaining reparametrization invariance}

For the type of discretized actions we discussed so far one can always define a discrete action which displays exact reparametrization invariance. This so-called perfect action reflects the gauge freedom of the continuous system, which results in a non-uniqueness of the solution $\left\{t_{n}, q_{n}\right\}$. The idea is that the discrete system should exactly reproduce the dynamics of the continuous system, determined by the continuum Lagrange function $L(q, \dot{q})$.

We define the perfect action as follows: For $t_{n}, q_{n}$, and for each $n=0, \ldots N-1$ solve the continuum Euler-Lagrange equations for $t^{(n)}(s), q^{(n)}(s), s \in[0,1]$ with boundary values

$$
\begin{aligned}
& t^{(n)}(0)=t_{n} \quad q^{(n)}(0)=q_{n} \\
& t^{(n)}(1)=t_{n+1} \quad q^{(n)}(1)=q_{n+1} .
\end{aligned}
$$

Denote the value of the action $S$ on that solution, which is nothing but the HamiltonJacobi functional, by $S_{H J}^{(n)}$ and define

$$
\begin{aligned}
S_{e} & :=\sum_{n=0}^{N-1} S_{H J}^{(n)}\left(t_{n}, q_{n}, t_{n+1}, q_{n+1}\right) \\
& =\sum_{n=0}^{N-1} \int_{0}^{1} d s \tilde{L}\left(t^{(n)}(s), q^{(n)}(s), t^{(n)^{\prime}}(s), q^{(n)^{\prime}}(s)\right)
\end{aligned}
$$

where $t^{(n)^{\prime}}$ and $q^{(n)^{\prime}}$ denote the derivatives of $t^{(n)}$ and $q^{(n)}$ w.r.t. the curve parameter $s$, respectively, and $\tilde{L}$ is given by (2.1).

The discrete action $S_{e}$ defined in (2.12) is exactly reparametrization invariant, as the following theorem shows.

Theorem: For each solution $\left\{t_{n}, q_{n}\right\}$ of the equations of motion determined by the action (2.12) and each sequence $\left\{s_{n}\right\}$ there is a solution $t(s), q(s)$ of the equations of motion (2.3), (2.4) with $t\left(s_{n}\right)=t_{n}, q\left(s_{n}\right)=q_{n}$. Furthermore, for every such solution $t(s), q(s)$

\footnotetext{
${ }^{1}$ Where, however, the broken symmetry in question is usually global Poincaré symmetry, and not gauge symmetries.
} 
and each $s_{0}<s_{1} \ldots<s_{N},\left\{t\left(s_{n}\right), q\left(s_{n}\right)\right\}$ is a solution to the equations of motion determined by (2.12).

Proof: A detailed proof of this can be found in [6].

Since the continuous system with the Lagrangian $\tilde{L}$ is reparametrization invariant, the solutions of (2.3), (2.4) are highly non-unique. Therefore, also the boundary value problem for the action (2.12) has a vast amount of different solutions for the same boundary conditions. This non-uniqueness directly corresponds to the reparametrization invariance of the action (2.2), and hence the discrete action $S_{e}$ exactly captures this invariance. In particular, the $t_{n}, q_{n}$ are underdetermined. Given the uniqueness of solutions to the dynamics determined by the deparametrized system with Lagrangian $L(q, \dot{q})$ - the $q_{n}$ are uniquely determined by the $t_{n}$, which by themselves can be chosen arbitrarily 2. It follows that there is one gauge degree of freedom per vertex. Note that the $q_{n}\left(t_{n}\right)$ are Dirac observables in the sense of [17].

We have seen that the discrete action (2.12) exactly mimics the continuum dynamics of the system and therefore exhibits exact reparametrization invariance, unlike the system defined by the naïve discretization (2.5). Note that, as the discretization becomes very fine, one can expect the system to be approximately reparametrization invariant in the sense of [7]. The Hessian of $S_{d}$ at the solution will contain a large number of Eigenvalues 3 approaching zero in the continuum limit, when reparametrization invariance is restored.

\subsection{Improving the discrete action $S_{d}$}

The perfect action $S_{e}$ contains the Hamilton-Jacobi functional of the system defined by the Lagrangian $L$, which might in general be hard to compute, or even unknown. In the following we present a procedure to construct sequences of improved actions, which converge to the perfect action, and which satisfy the constraints in an approximate way [7].

In order to improve the action $S_{d}$, which is a naïve discretization of the action (2.2) on the discretized interval $\left\{t_{n}\right\}$, one needs to refine the interval by $t_{n}=\tilde{t}_{n M}<\tilde{t}_{n M+1}<$ $\ldots<\tilde{t}_{n M+(M-1)}<\tilde{t}_{(n+1) M}=t_{n+1}$. Fix $\left\{t_{n}, q_{n}\right\}$, and for each interval $\left[t_{n}, t_{n+1}\right]$ solve the discrete equations of motion or the $\tilde{t}_{k}, \tilde{q}_{k}$, given by the naïve discretization of the action $S$, i.e. find an extremum of the action

$$
S_{d}^{(n)}=\sum_{k=M n}^{M n+M-1} L\left(\tilde{q}_{k}, \frac{\tilde{q}_{k+1}-\tilde{q}_{k}}{\tilde{t}_{k+1}-\tilde{t}_{k}}\right)\left(\tilde{t}_{k+1}-\tilde{t}_{k}\right)
$$

with the boundary conditions

\footnotetext{
${ }^{2}$ As long as $t_{n}<t_{n+1}$ for all $n$, i.e. the $t_{n}$ are a growing sequence.

${ }^{3}$ Namely one per (inner) vertex.
} 


$$
\begin{aligned}
\tilde{t}_{M n} & =t_{n}, \quad \tilde{q}_{M n}=q_{n} \\
\tilde{t}_{M(n+1)} & =t_{n+1}, \quad \tilde{q}_{M(n+1)}=q_{n+1} .
\end{aligned}
$$

Denote the value of $S_{d}^{(n)}$ on the solution by $S_{*}^{(n)}$. Then the action

$$
S_{*}:=\sum_{n=0}^{N-1} S_{*}^{(n)}
$$

is clearly a function of the chosen $t_{n}, q_{n}$. It is more complicated than the naïve discretization (2.5).

Since for very fine subdivision the $\tilde{t}_{k}, \tilde{q}_{k}$ converge to a solution $t(s), q(s)$ of the continuum dynamics given by $\tilde{L}$, it is easy to see that - in the limit of very fine discretization $\tilde{t}_{k}$ each of the contributions $S_{*}^{(n)}$ converges to its continuous counterpart, i.e.

$$
\lim _{M \rightarrow \infty} S_{*}^{(n)}=S_{H J}^{(n)}\left(t_{n}, q_{n}, t_{n+1}, q_{n+1}\right)=\int_{s_{n}}^{s_{n+1}} d s L(t(s), q(s))
$$

Therefore $S_{*}$ converges to the exact discrete action (2.12).

The naïvely discretized action (2.5) approximates the exact discrete action (2.12) by replacing, for each interval $\left[s_{n}, s_{n+1}\right]$, the integral over the Lagrangian by a Riemann sum involving only two points. The improvement within the action $S_{*}$ lies in the fact that the Riemann sum used to approximate the integral relies on many more intermediate points, therefore delivering a better approximation.

In order to compute the improved actions, only the solutions to the naïvely discretized action $S_{d}$ for a refined discretization is involved, making the computation possibly more feasible, if the continuum system is not at hand, or too difficult to solve. Furthermore, the improved action $S_{*}$ can can be made an arbitrarily good approximation to the exact discrete action $S_{e}$, by using a very fine discretization, or by iterating the process, i.e. computing $S_{*},\left(S_{*}\right)_{*},\left(\left(S_{*}\right)_{*}\right)_{*}, \ldots$, which lead to the same limit $S_{e}$. It can therefore be used to compute $S_{e}$ recursively, which can therefore be seen as the "perfect limit" of the $S_{*}$. We will use this strategy in order to investigate the perfect action in Regge gravity later on.

Note that although $S_{*}$ still does not retain the full reparametrization invariance of $S_{e}$, it is closer to it than the naïvely discretized action $S_{d}$, in the sense that the constraints are satisfied to a greater accuracy.

\footnotetext{
${ }^{4}$ See [] for details on approximate constraints.
} 


\section{Regge Calculus}

In the previous system we have seen that one-dimensional reparametrization invariant systems usually lose that invariance after discretization. This is also true for higherdimensional field theories: Classical GR, as a theory of metrics on a differential manifold, is reparametrization invariant, due to the principle of covariance [1]. It is this invariance which makes it very difficult to compute, or interpret the physics of solutions. It also is connected to many obstacles for quantizing the theory [푸, 8, 18, 19].

Regge Calculus provides a discretization of GR, by triangulating the manifold, and replacing curvature expressions with deficit angles around 2-codimensional subsimplices [2]. Just as the discretization of one-dimensional systems replaces the search for smooth solutions to the equations by piecewise linear ones, Regge calculus replaces smooth curved metrics by piecewise linear flat ones.

\subsection{Continuous preliminaries}

The Einstein-Hilbert action in $D$ dimensions with cosmological constant $\Lambda$ is given by

$$
S_{E H}=\frac{1}{8 \pi} \int_{\mathcal{M}} d^{D} x \sqrt{|g|}\left(\Lambda-\frac{1}{2} R\right)
$$

leading to the equations of motion

$$
8 \pi \frac{\partial S_{E H}}{\partial g^{\mu \nu}}=R_{\mu \nu}-\frac{1}{2} g_{\mu \nu} R+\Lambda g_{\mu \nu}=0
$$

There is a special solution to (3.1), which will be very important later on. The Riemann tensor of a space of constant (sectional) curvature $\kappa$ has the property

$$
R_{\mu \nu \sigma \rho}=\kappa\left(g_{\mu \rho} g_{\nu \sigma}-g_{\mu \sigma} g_{\nu \rho}\right)
$$

leading to $R_{\mu \nu}=\kappa(D-1) g_{\mu \nu}$ and $R=\kappa D(D-1)$. Therefore the metric satisfying (3.2) satisfies the equations (3.1) for

$$
\Lambda=\frac{(D-1)(D-2)}{2} \kappa
$$

\subsection{Discrete action}

In Regge calculus, the smooth manifold $\mathcal{M}$ is replaced by a triangulated manifold $\mathcal{T}$, the $D$-simplices of which are internally flat [2]. The Riemann curvature in this now arises as nontrivial parallel transport resulting from the nontrivial way of gluing the simplices together. The curvature is therefore naturally associated to the $D-2$ simplices $H$ (also called "hinges") in the complex.

The Ricci scalar for such a manifold is 2 times the deficit angle at a $D-2$ simplex. If the triangulation $\mathcal{T}$ has a boundary $\partial \mathcal{T}$ (which is a triangulated $D$-1-dimensional manifold), then the action has a contribution from the extrinsic curvature in the boundary and the Ricci curvature in the bulk $\mathcal{T}^{\circ}:=\mathcal{T} \backslash \partial \mathcal{T}$, and reads 5 [20]

\footnotetext{
${ }^{5} \mathrm{Up}$ to a factor of $8 \pi$, which we ignore from now on.
} 


$$
S_{\mathcal{T}}=\sum_{h \in \mathcal{T}^{\circ}} F_{h} \epsilon_{h}-\Lambda \sum_{\sigma \subset \mathcal{T}^{\circ}} V_{\sigma}+\sum_{h \in \partial \mathcal{T}} F_{h} \psi_{h}
$$

The sum goes over all $(D-2)$-simplices $h$ in the bulk and the boundary separately. The associated angles are

$$
\begin{array}{ll}
\epsilon_{h}=2 \pi-\sum_{\sigma \supset h} \theta_{h}^{\sigma} \quad \text { for } h \in \mathcal{T}^{\circ} \\
\psi_{h}=\pi-\sum_{\sigma \supset h} \theta_{h}^{\sigma} \quad \text { for } h \in \partial \mathcal{T}
\end{array}
$$

where the $\theta_{h}^{\sigma}$ is the interior dihedral angle in the $D$-simplex $\sigma$ associated to the $D-2$ subsimplex $h \subset \sigma$. For these angles within a flat simplex $\sigma$ the so-called Schlaefli-identity reads

$$
\sum_{h \subset \sigma} F_{h} \frac{\partial \theta_{h}^{\sigma}}{\partial l_{e}}=0 \quad \text { for all 1-simplices ("edges") } e \subset \sigma
$$

The dynamical variables are taken to be the lengths $l_{e}$ of the edges $e \in \mathcal{T}^{\circ}$ in the bulk. For those edges the equations of motion can be computed with (3.7) to be:

$$
\sum_{h \supset e} \frac{\partial F_{h}}{\partial l_{e}} \epsilon_{h}-\Lambda \sum_{\sigma \supset e} \frac{\partial V_{\sigma}}{\partial l_{e}}=0
$$

Instead of piecewise linear flat simplices, one can build up the triangulation with simplices of constant (sectional) curvature $\kappa$ (see appendix A). The Regge action for such a triangulation $\mathcal{T}$ with cosmological constant $\Lambda$ is a sum of the overall curvature of the manifold, having a contribution from the deficit angles at the $D-2$ dimensional subsimplices, the constant curvature of the tetrahedra, and the term with the cosmological constant. For $\Lambda$ and $\kappa$ having the relation (3.3), this leads to

$$
S_{\mathcal{T}}^{(\kappa)}=\sum_{h \subset \mathcal{T}^{\circ}} F_{h}^{(\kappa)} \epsilon_{h}^{(\kappa)}+(D-1) \kappa \sum_{\sigma \subset \mathcal{T}^{\circ}} V_{\sigma}^{(\kappa)}+\sum_{h \in \partial \mathcal{T}} F_{h}^{(\kappa)} \psi_{h}^{(\kappa)} .
$$

where $F_{h}^{(\kappa)}$ denotes the $D$-2-dimensional volume of the $D-2$ simplex $h \subset \sigma$. Furthermore $\epsilon_{h}^{(\kappa)}$ and $\psi_{h}^{(\kappa)}$ denote deficit angle and exterior angle in the curved simplices analogously to $(3.5)$, (3.6).

The Schlaefli identity (A.5) for curved simplices leads to the equations

$$
\frac{\partial S_{\mathcal{T}}^{(\kappa)}}{\partial l_{e}}=\sum_{h \supset e} \frac{\partial F_{h}^{(\kappa)}}{\partial l_{e}} \epsilon_{h}^{(\kappa)}=0
$$

\subsection{Gauge invariance in Regge Calculus}

Analogously to our observations in the last chapter, the reparametrization invariance of General Relativity is lost in Regge Calculus, in the following sense: For a given set of 
boundary lengths, the solutions to Regge's equations (3.8) are generically unique, i.e. completely determined by the boundary data. The only exceptions to this are the cases in which the discrete dynamics exactly reproduces the continuum dynamics.

In $3 D$ with $\Lambda=0$ the Regge equations (3.8) are simply the vanishing of the deficit angles $\epsilon_{e}=0$, the solution of which is a triangulation of a locally flat space-time. This is also the solution to $3 D \mathrm{GR}$ with vanishing cosmological constant. In higher dimensions there is, among other solutions, also $\epsilon_{h}=0$, which can readily be seen to solve (3.8) for $\Lambda=0$. Again, this coincides with locally flat space-time which is also one (among many solutions) of GR for $D>3$.

In all of these cases the solutions possess a vertex displacement symmetry and an invariance under Pachner moves, which in $3 D$ can e.g. be seen as a result of the second Bianchi identities [14, 15]. As a result, the bulk lengths $l_{e}, e \in \mathcal{T}^{\circ}$ are not uniquely determined by the boundary lengths $l_{e}, e \in \partial \mathcal{T}$, rather the vertex displacement symmetry results in $D$ gauge degrees of freedom per vertex.

Apart from these special cases, where the discrete dynamics exactly reproduces the continuum dynamics, the boundary data fixes uniquely the lengths of the edges in the interior of the triangulation [7].6 That is translating a vertex in a solution does not lead to another solution (as it does for $3 D$ Regge calculus with $\Lambda=0$ ) and the Hamilton-Jacobi functional, i.e. the action evaluated on a solution, is not invariant under Pachner moves of the bulk. This is analogous to the situation in one dimension, where the reparametrization invariance (which in discretized gravity would amount to an invariance under change of triangulation) is lost in the naïve discretization.7

\section{Improved and perfect action in $3 D$}

Since lattice gauge theory is not diffeomorphism-invariant, the symmetries that are broken by discretization are not its local gauge symmetries, which are of a different nature than in GR, but Poincaré-invariance. The methods to construct improved and perfect actions in QCD can therefore not be directly transferred to GR. We therefore attempt to generalize the way this is done for one-dimensional systems, encountered in chapter 2, to the case of Regge Calculus.

In one dimension the interval, on which the continuous theory is defined, is divided into smaller intervals as a result of the discretization, and in order to define the improved action (2.14) the interiors of these intervals are then further refined. The discrete equations are then solved for the refined lattice, subject to boundary conditions which relate them to values on the coarse lattice. Therefore, since in Regge Calculus spacetime is split into simplices via a triangulation, we will refine this triangulation further into smaller simplices in order to improve the action. Note that in more than one dimension the boundary of a triangulation and between single simplices is nontrivial, and it needs to be refined as well.

\footnotetext{
${ }^{6}$ Apart from discrete ambiguities, which we ignore for the time being [16, 7].

${ }^{7}$ The exceptions for this, e.g. $3 D$ with $\Lambda=0$, can be compared to the free particle in one dimension, where the continuum solutions are linear dependencies between the $t_{n}$ and the $q_{n}$. In fact, the naïve discretization (2.5) already coincides with the perfect action (2.12) for this case, and the solutions are not uniquely determined by the boundary data $t_{0}, t_{N}, q_{0}, q_{N}$. Rather, the Hessian has as many zero Eigenvalues as inner vertices and the solutions $t_{n}, q_{n}$ are non-unique in the sense of chapter 2.1 which is a reflection of the gauge symmetry of the continuum limit in this case.
} 


\subsection{Refinement of the Regge action}

We will first demonstrate the procedure for $D=3$ to show the general idea, before we turn to the case of higher dimensions (in particular $D=4$, which is the case of most interest to us) in chapter 5.

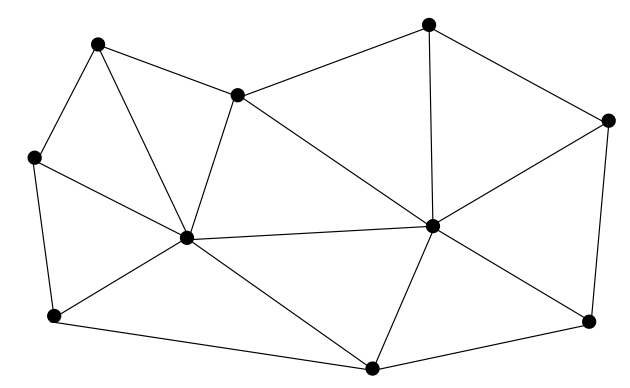

Figure 1: Coarse triangulation $\mathcal{T}$ consisting of edged $E$, triangles $T$ and tetrahedra $\Sigma$.

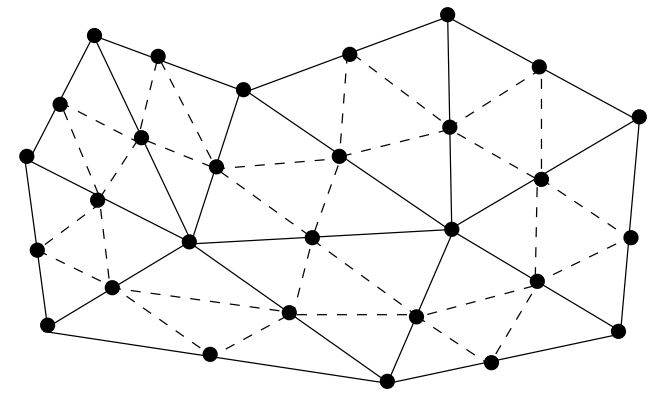

Figure 2: Fine triangulation $\tau$ consisting of edged $e$, triangles $t$ and tetrahedra $\sigma$.

Consider a three-dimensional triangulation $\mathcal{T}$, consisting of edges $E$, triangles $T$ and tetrahedra $\Sigma$, possibly with a boundary $\partial \mathcal{T}$. The Regge action $S_{\mathcal{T}}$ is given by (3.4), and is a function of the edge lengths $L_{E}$. Now subdivide $\mathcal{T}$ into a finer triangulation $\tau$, consisting of edges $e$, triangles $t$ and tetrahedra $\sigma$. Similarly to the definition of the improved action in $1 D$, we solve the Regge equations for the edge lengths $l_{e}$ subject to the conditions

$$
\sum_{e \subset E} l_{e}=L_{E}
$$

and define the improved action $S_{\mathcal{T}, \tau}$ as the value of the Regge action $S_{\tau}$ on a solution of the equations for $l_{e}$ subject to (4.1). We add the constraint (4.1) via Lagrange multipliers, i.e. we have to vary the action

$$
S_{\tau}=\sum_{e} l_{e} \varphi_{e}-\Lambda \sum_{\sigma} V_{\sigma}+\sum_{E} \alpha_{E}\left(L_{E}-\sum_{e \subset E} l_{e}\right)
$$

where we have defined $\varphi_{e}:=\psi_{e}$ for $e \in \partial \tau$ and $\varphi_{e}:=\epsilon_{e}$ for $e \in \tau^{\circ}$, to unify notation. The equations of motion are then given by deriving (4.2) w.r.t the $l_{e}$ and $\alpha_{E}$, i.e. one gets

$$
\begin{aligned}
\frac{\partial S_{\tau}}{\partial l_{e}} & =\varphi_{e}-\Lambda \sum_{\sigma \supset e} \frac{\partial V_{\sigma}}{\partial l_{e}}-\sum_{E \supset e} \alpha_{E}=0 \\
\frac{\partial S_{\tau}}{\partial \alpha_{E}} & =L_{E}-\sum_{e \subset E} l_{e}=0 .
\end{aligned}
$$

The improved action is then defined as the value of $S_{\tau}$ on a solution of (4.3), (4.4), i.e.

$$
S_{\mathcal{T}, \tau}:=\left.S_{\tau}\right|_{\frac{\partial S_{\tau}}{\partial l_{e}}=\frac{\partial S_{\tau}}{\partial \alpha_{E}}=0}
$$


Note that the improved action $S_{\mathcal{T}, \tau}$ depends on the "large" lengths $L_{E}$ for $E \in \mathcal{T}$, but incorporates the dynamics of the finer triangulation $\tau$. A quick calculation using Euler's theorem (A.6) shows that the $l_{e}, \alpha_{E}$ satisfy

$$
0=\sum_{e} l_{e} \frac{\partial S_{\tau}}{\partial l_{e}}=\sum_{e} l_{e} \varphi_{e}-3 \Lambda \sum_{\sigma} V_{\sigma}-\sum_{E} \alpha_{E} \sum_{e \subset E} l_{e} .
$$

So, with (4.4) the improved action can be put into the form

$$
S_{\mathcal{T}, \tau}=\sum_{E} L_{E} \alpha_{E}+2 \Lambda \sum_{\Sigma} V_{\Sigma}
$$

where we have defined $V_{\Sigma}:=\sum_{\sigma \subset \Sigma} V_{\sigma}$. Note that the $\alpha_{E}, V_{\Sigma}$ are complicated functions of the $L_{E}$, which have to be determined by the equations of motion (4.3), (4.4). Nevertheless, one can derive the equations of motion by varying $S_{\mathcal{T}, \tau}$ w.r.t. the $L_{E}$. This can be achieved by changing the $L_{E} \rightarrow L_{E}+\delta L_{E}$, and assuming that the solutions for the $l_{e}$ and $\alpha_{E}$ also change only slightly to $l_{e} \rightarrow l_{e}+\delta l_{e}, \alpha_{E} \rightarrow \alpha_{E}+\delta \alpha_{E}$. Therefore the value of $S_{\mathcal{T}, \tau}$ changes by

$$
\begin{aligned}
\delta S_{\mathcal{T}, \tau} & =\sum_{e} \frac{\partial S_{\tau}}{\partial l_{e}} \delta l_{e}+\sum_{E} \frac{\partial S_{\tau}}{\partial \alpha_{E}} \delta \alpha_{E}+\left.\sum_{E} \frac{\partial S_{\tau}}{\partial L_{E}} \delta L_{E}\right|_{\frac{\partial S_{\tau}}{\partial l_{e}}=\frac{\partial S_{\tau}}{\partial \alpha_{E}}=0} \\
& =\alpha_{E} \delta L_{E} .
\end{aligned}
$$

Therefore the equations for the $L_{E}$ determined by the improved action $S_{\mathcal{T}, \tau}$ are the vanishing of the Lagrange multipliers, i.e.

$$
\frac{\partial S_{\mathcal{T}, \tau}}{\partial L_{E}}=\alpha_{E} \stackrel{!}{=} 0 \quad \text { for } E \in \mathcal{T}^{\circ}
$$

which, together with (4.3) are equivalent to the Regge equations for the $l_{6}^{8}$.

\subsection{Perfect action in $3 D$}

There is a similarity between the improved action (4.7) and the Regge action with curved simplices (3.9), as well as the respective resulting equations of motion (4.9) and (3.10). The similarity becomes more apparent if we define

$$
\Theta_{E}^{\Sigma}:=\sum_{\sigma \supset e, \sigma \in \Sigma}\left(\theta_{e}^{\sigma}-\Lambda \frac{\partial V_{\sigma}}{\partial l_{e}}\right)
$$

for some $9 \subset E$, then we have $\alpha_{E}=2 \pi-\sum_{\Sigma \supset E} \Theta_{E}^{\Sigma}$ for $E \subset \mathcal{T}^{\circ}$ being in the bulk and $\alpha_{E}=\pi-\sum_{\Sigma \supset E} \Theta_{E}^{\Sigma}$ for $E \subset \partial \mathcal{T}$ being in the boundary. For every edge $E \subset \mathcal{T}^{\circ}$ in the bulk, the equations of motion determined by $S_{\mathcal{T}, \tau}$ therefore are

$$
2 \pi-\sum_{\Sigma \supset E} \Theta_{E}^{\Sigma}=0
$$

\footnotetext{
${ }^{8}$ To be precise: It is equivalent to $\frac{\partial S_{\tau}}{\partial l_{e}}=0$ for all bulk edges $e \in \tau^{\circ}$, all boundary edges $e \in \partial \tau$ which are not a subedge of an edge in $\partial \mathcal{T}$, i.e. $e \nsubseteq E$, and (4.3) for all $e \in \partial \tau$ which are, i.e. also $e \subset E \in \partial \mathcal{T}$. This is equivalent to the Regge equations for the $e$ in the finer triangulation $\tau$, plus the vanishing of the canonical momenta on the boundary triangles $T \in \partial \mathcal{T}$.

${ }^{9}$ The equations (4.3) guarantee that this choice does not depend on the actual $e \subset E$.
} 
Note that, despite the formal similarity, the $\Theta_{E}^{\Sigma}$ are not quite the interior dihedral angles at the edges $E$ in the tetrahedra $\Sigma$. It is, however, not hard to show that they become so in the perfect limit, i.e. the limit of infinitely fine subdivision, which we denote by $\tau \rightarrow \infty$. If the triangulation $\tau$ is such that its simplices $\sigma$ are regular, i.e. their edge lengths $l_{e}$ after solving (4.3), (4.4) are all of the same small order of magnitude $\lambda$, then the term in (4.10) containing the derivative of the volume scales like $O\left(\lambda^{2}\right)$, as compared to the $\theta_{e}^{\sigma}$, which scale as $O(1)$, and therefore dominate the expression. (Also note that $\theta_{e}^{\sigma}-\kappa \frac{\partial V_{\sigma}}{\partial l_{e}}$ is the first order Taylor expansion in $\kappa$ of the dihedral angle in a curved tetrahedron.) We conclude that, in the perfect limit $\tau \rightarrow \infty$ the $\Theta_{E}^{\Sigma}$ indeed converge to the sum of the interior angles at $e \subset E$ in $\sigma \subset \Sigma$, i.e. to the interior angle at $E$ in $\Sigma$. Note that this interior angle is the same everywhere "on" $E$, since it is independent of $e \subset E$. Even more, we can demonstrate the perfect limit of $V_{\Sigma}$ (as a function of the $L_{E}$ ) by e.g. considering a triangulation $\tau$ consisting of only one tetrahedron $|\mathcal{T}|=\Sigma$. Then the variations (4.9) of $S_{\mathcal{T}, \tau}$ with respect to one of the $L_{E}, E=1, \ldots, 6$ is equivalent to

$$
\sum_{E=1}^{6} L_{E} \frac{\partial \Theta_{E}}{\partial L_{E}}=2 \Lambda \frac{\partial V_{\Sigma}}{\partial L_{E}}
$$

which, in the perfect limit, is exactly the Schlaefli identity for curved tetrahedra (A.5) which related the interior angles of curved dihedral angles and volumes on tetrahedra of constant curvature $\kappa=\Lambda$. In the perfect limit, the formal similarity becomes an equality, and we conclude that the perfect action in $3 D$ is given by

$$
S_{\mathcal{T}, *}:=\lim _{\tau \rightarrow \infty} S_{\mathcal{T}, \tau}=S_{\mathcal{T}}^{(\kappa)},
$$

i.e. coincides with the Regge action for constantly curved tetrahedra with curvature $\kappa=\Lambda$. It is quite easy to show that this action has three gauge degrees of freedom per vertex, unlike $S_{\mathcal{T}}$, since the equations of motion - given by the perfect limit of (4.11) - are equivalent to the vanishing of all deficit angles $\epsilon_{E}=0$ for interior edges $E \in \mathcal{T}^{\circ}$, which results in the triangulation of a manifold of constant sectional curvature $\kappa=\Lambda$. This not only reproduces exactly the continuum dynamics of $3 D$ GR with cosmological constant $\Lambda$, but also posesses the exact vertex displacement symmetry as $3 D$ Regge calculus with flat simplices exhibits for $\Lambda=0$. Furthermore, it is invariant under refinement of triangulation $\mathcal{T}$, as it should be by construction.

We conclude that in $3 D$, the gauge symmetry of $G R$ containing 3 gauge degrees of freedom per vertex, which is broken for $\Lambda \neq 0$, is restored in the perfect limit. The Regge action for constantly curved tetrahedra arises naturally as perfect action in this context. It should be noted that the Regge action (3.4) with flat simplices arises naturally as first order approximation, by the following argument: By investigating the scaling property of the curved Regge action (3.9), e.g. by considering (A.7), one can easily see that a scaling of the edge lengths $l_{e} \rightarrow \lambda l_{e}$ can be absorbed into a scaling of the curvature $\kappa \rightarrow \lambda^{2} \kappa$. Expanding the curved functions $\theta_{e}^{(\kappa), \sigma}, V_{\sigma}^{(\kappa)}$ into linear order in $\kappa$, one obtains, by using the identities (A.4) and (A.5), that

$$
S_{\tau}^{(\kappa)}=S_{\tau}+O\left(\kappa^{2}\right)
$$

where $S_{\tau}$ is the Regge action (3.4) for flat simplices with cosmological constant $\Lambda=\kappa$. 


\section{$5 \quad$ Higher dimensions}

We now consider the concept of improved and perfect actions for dimensions $D>3$, where of course the case of ultimate interest is $D=4$. Nevertheless, since the arising procedures are generic for arbitrary higher dimensions, we shall treat the problem for arbitrary dimension $D$, and comment about the implications for $D=4$ in the end.

The general concept for defining the improved action $S_{\mathcal{T}, \tau}$ for $D>3$ is similar to $D=3$. We start with a triangulation $\mathcal{T}$ consisting of simplices $\Sigma$, hinges $H$ and edges $E$. Now subdivide $\mathcal{T}$ into a finer triangulation $\tau$, consisting of $D$-simplices $\sigma$, hinges $h$ and edges $e$. Note that some of the hinges $h$ are contained in the "larger" hinges $H$. The action for the finer triangulation $S_{\tau}$ is a function of the edge lengths $l_{e}$. It turns out that the most convenient generalization of the condition (4.1) to $D>3$ is not to keep the edge

lengths $L_{E}$ fixed, but rather the $D-2$-volumes $F_{H}$, i.e. to constrain the variation of the Regge action for $\tau$ by

$$
\sum_{h \subset H} f_{h}=F_{H}
$$

where $f_{h}$ is the $D-2$-volume of the hinge $h$. In other words, we vary

$$
S_{\tau}=\sum_{h} f_{h} \varphi_{h}-\Lambda \sum_{\sigma} V_{\sigma}+\sum_{H} \alpha_{H}\left(F_{H}-\sum_{h \subset H} f_{h}\right)
$$

with respect to $l_{e}$ and $\alpha_{H}$, where the Lagrange multipliers $\alpha_{H}$ have been introduced in order to enforce (5.1), and $\varphi_{h}$ denotes the deficit angle $\epsilon_{h}$ for $h \in \tau^{\circ}$ being in the bulk, and the extrinsic curvature angle $\psi_{h}$ for $h \in \partial \tau$ in the boundary. The improved action is - similar as for $D=3$ - defined as

$$
S_{\mathcal{T}, \tau}:=\left.S_{\tau}\right|_{\frac{\partial S_{\tau}}{\partial l_{e}}=\frac{\partial S_{\tau}}{\partial \alpha_{H}}=0}
$$

and is naturally a function of the $F_{H}$ (e.g. the areas of the triangles for $D=4$.). The resulting equations for the $l_{e}, \alpha_{H}$ are, using the Schlaefli-identity (3.7)

$$
\begin{aligned}
\frac{\partial S_{\tau}}{\partial l_{e}} & =\sum_{h \supset e} \frac{\partial f_{h}}{\partial l_{e}} \varphi_{h}-\Lambda \sum_{\sigma \supset e} \frac{\partial V_{\sigma}}{\partial l_{e}}-\sum_{h \supset e} \sum_{H \supset h} \alpha_{H} \frac{\partial f_{h}}{\partial l_{e}}=0, \\
\frac{\partial S_{\tau}}{\partial \alpha_{H}} & =F_{H}-\sum_{h \subset H} f_{h}=0 .
\end{aligned}
$$

Using Euler's theorem (A.6) we get

$$
0=\sum_{e} l_{e} \frac{\partial S_{\tau}}{\partial l_{e}}=(D-2) \sum_{h} f_{h} \varphi_{h}-D \Lambda \sum_{\sigma} V_{\sigma}-(D-2) \sum_{H} \alpha_{H} \sum_{h \subset H} f_{h},
$$

which, inserted into (5.2) together with (5.5), results in the improved action

$$
S_{*}=\sum_{H} F_{H} \alpha_{H}+\frac{2}{D-2} \Lambda \sum_{\Sigma} V_{\Sigma},
$$


where we have defined $V_{\Sigma}:=\sum_{\sigma \subset \Sigma} V_{\sigma}$. Note the similarity between the improved action (5.7) and the Regge action (3.9) for simplices of constant curvature $\kappa$, for $\kappa$ and $\Lambda$ being related by (3.3).

The improved action (5.7) is a function of the $F_{H}$ via the $\alpha_{H}$ and $V_{\Sigma}$, which will depend on the $F_{H}$ in a complicated manner to be determined by solving the equations (5.4), (5.5). Nevertheless, we can derive the equations for the $F_{H}$ determined by the improved action. For this we consider the same set of equations, just with slightly changed parameters $F_{H}+\delta F_{H}$. It can be expected that the solutions for $l_{e}, \alpha_{H}$ will also change just slightly via

$$
\begin{gathered}
l_{e} \longrightarrow l_{e}+\delta l_{e} \\
\alpha_{H} \longrightarrow \alpha_{H}+\delta \alpha_{H}
\end{gathered}
$$

Then $S_{\mathcal{T}, \tau}$ changes slightly via

$$
\delta S_{\mathcal{T}, \tau}=\sum_{e} \frac{\partial S_{\tau}}{\partial l_{e}} \delta l_{e}+\sum_{H} \frac{\partial S_{\tau}}{\partial \alpha_{H}} \delta \alpha_{H}+\sum_{H} \frac{\partial S_{\tau}}{\partial F_{H}} \delta F_{H},
$$

and evaluating (5.8) on a solution results in

$$
\frac{\partial S_{\mathcal{T}, \tau}}{\partial F_{H}}=\alpha_{H}=0
$$

\subsection{Improving the curved Regge action}

It is instructive to repeat the calculation with curved simplices. We start from the action (3.9) and impose the constraints via Lagrange multipliers $\alpha_{H}^{(\kappa)}$. In other words, we have to vary the action

$$
S_{\tau}^{(\kappa)}=\sum_{h} f_{h}^{(\kappa)} \varphi_{h}^{(\kappa)}+(D-1) \kappa \sum_{\sigma} V_{\sigma}^{(\kappa)}+\sum_{H} \alpha_{H}^{(\kappa)}\left(F_{H}-\sum_{h \subset H} f_{h}^{(\kappa)}\right)
$$

where the superscript $\left.{ }^{(} \kappa\right)$ denotes the volume of hinges and simplices of constant curvature $\kappa$. Again, $\varphi_{h}^{(\kappa)}$ is shorthand for $\epsilon_{h}^{(\kappa)}$ whenever $h \in \tau^{\circ}$ is a hinge in the bulk, and $\psi_{h}^{(\kappa)}$, whenever $h \in \partial \tau$ is in the boundary. With the Schlaefli-identity (A.5) for simplices of constant curvature, the resulting equations for the $l_{e}$ are

$$
\begin{aligned}
\frac{\partial S_{\tau}^{(\kappa)}}{\partial l_{e}} & =\sum_{h \supset e} \frac{\partial f_{h}^{(\kappa)}}{\partial l_{e}} \varphi_{h}^{(\kappa)}-\sum_{h \supset e} \sum_{H \supset h} \alpha_{H}^{(\kappa)} \frac{\partial f_{h}^{(\kappa)}}{\partial l_{e}}=0 \\
\frac{\partial S_{\tau}^{(\kappa)}}{\partial \alpha_{H}^{(\kappa)}} & =F_{H}-\sum_{h \subset H} f_{h}^{(\kappa)}=0
\end{aligned}
$$

With the geometric identity (A.9) for simplices of constant curvature $\kappa$, we get

$$
\begin{aligned}
0=\sum_{e} l_{e} \frac{\partial S_{\tau}^{(\kappa)}}{\partial l_{e}}= & (D-2) \sum_{h} f_{h}^{(\kappa)} \varphi_{h}^{(\kappa)}+2 \kappa \sum_{h} \frac{\partial f_{h}^{(\kappa)}}{\partial \kappa} \varphi_{h}^{(\kappa)} \\
& -(D-2) \sum_{H} \sum_{h \subset H} \alpha_{H}^{(\kappa)} f_{h}^{(\kappa)}-2 \kappa \sum_{H} \sum_{h \subset H} \alpha_{H}^{(\kappa)} \frac{\partial f_{h}^{(\kappa)}}{\partial \kappa}
\end{aligned}
$$


which results in the improved action

$$
\begin{aligned}
S_{\mathcal{T}, \tau}^{(\kappa)}= & \sum_{H} F_{H} \alpha_{H}^{(\kappa)}+(D-1) \kappa \sum_{\Sigma} V_{\Sigma}^{(\kappa)} \\
& +\frac{2}{D-2} \kappa \sum_{h} \frac{\partial f_{h}^{(\kappa)}}{\partial \kappa} \varphi_{h}^{(\kappa)}+\frac{2}{D-2} \kappa \sum_{H} \sum_{h \subset H} \alpha_{H}^{(\kappa)} \frac{\partial f_{h}^{(\kappa)}}{\partial \kappa}
\end{aligned}
$$

where we have defined $V_{\Sigma}^{(\kappa)}:=\sum_{\sigma \subset \Sigma} V_{\sigma}^{(\kappa)}$. By a similar reasoning as in the case with flat simplices, the equations for the improved action is easily obtained to be

$$
\frac{\partial S_{\mathcal{T}, \tau}^{(\kappa)}}{\partial F_{H}}=\alpha_{H}^{(\kappa)}=0
$$

\subsection{Perfect actions with flat and curved simplices}

If we consider the improved actions (5.7) and (5.14), which result from refining the triangulations with flat and curved simplices, respectively, we see that their expressions seem to be quite different. However, in performing the continuum limit for both actions, we will demonstrate that they both converge to the same perfect action, when $\Lambda$ and $\kappa$ satisfy the relation (3.3). In order to do this, we show that - as functions of the lengths $F_{H}$ - both perfect limits satisfy the same ordinary differential equation w.r.t. $\Lambda$ (or, equivalently, $\kappa)$. We do this by considering the ODE's that the two improved actions (5.7) and (5.14) satisfy, and show that in the continuum limit they converge to each other.

We first vary the improved action $S_{\mathcal{T}, \tau}$ for flat simplices w.r.t $\Lambda$, by solving the equations of motion again with $\Lambda \rightarrow \Lambda+\delta \Lambda$, and assume the resulting solutions $l_{e}, \alpha_{H}$ also change only slightly by $l_{e} \rightarrow l_{e}+\delta l_{e}$ and $\alpha_{H} \rightarrow \alpha_{H}+\delta \alpha_{H}$. The change of the action is therefore

$$
\begin{aligned}
\delta S_{\mathcal{T}, \tau} & =\sum_{e} \frac{\partial S_{\tau}}{\partial l_{e}} \delta l_{e}+\sum_{H} \frac{\partial S_{\tau}}{\partial \alpha_{H}} \delta \alpha_{H}+\frac{\partial S_{\tau}}{\partial \Lambda} \delta \Lambda \\
& =-\sum_{\Sigma} V_{\Sigma} \delta \Lambda,
\end{aligned}
$$

where the Regge equations have been used. With (5.7) this results in

$$
S_{\mathcal{T}, \tau}+\frac{2}{D-2} \Lambda \frac{\partial S_{\mathcal{T}, \tau}}{\partial \Lambda}=\sum_{H} F_{H} \alpha_{H} .
$$

The same calculation for the improved action (5.14) with curved simplices is more involved, since the constituents depend explicitly on $\kappa$. Since $S_{\mathcal{T}, \tau}^{(\kappa)}$ is the value of $S_{\tau}^{(\kappa)}$ evaluated on a solution, varying $S_{\mathcal{T}, \tau}^{(\kappa)}$ w.r.t $\kappa$ is equivalent to varying $S_{\tau}^{(\kappa)}$, and inserting the solutions for $l_{e}, \alpha_{H}^{(\kappa)}$ afterwards (since the variations of $l_{e}, \alpha_{H}^{(\kappa)}$ vanish on solutions, by definition). We have

$$
\begin{aligned}
\frac{\partial S_{\mathcal{T}, \tau}^{(\kappa)}}{\partial \kappa}= & \frac{\partial S_{\tau}^{(\kappa)}}{\partial \kappa}=\sum_{h} \frac{\partial f_{h}^{(\kappa)}}{\partial \kappa} \varphi_{h}^{(\kappa)}+\sum_{h} f_{h}^{(\kappa)} \frac{\partial \epsilon_{h}^{(\kappa)}}{\partial \kappa}+(D-1) \sum_{\sigma} V_{\sigma}^{(\kappa)} \\
& +(D-1) \kappa \sum_{\sigma} \frac{\partial V_{\sigma}^{(\kappa)}}{\partial \kappa}-\sum_{H} \sum_{h \subset H} \alpha_{H}^{(\kappa)} \frac{\partial f_{h}^{(\kappa)}}{\partial \kappa}
\end{aligned}
$$


With (A.4) and the Schlaefli identity (A.5), we have

$$
\sum_{h} f_{h}^{(\kappa)} \frac{\varphi_{h}^{(\kappa)}}{\partial \kappa}=-\frac{D-1}{2} \sum_{\sigma} \sum_{e \subset \sigma} l_{e} \frac{\partial V_{\sigma}^{(\kappa)}}{\partial l_{e}}
$$

which results in

$$
S_{\tau}^{(\kappa)}+\frac{2}{D-2} \kappa \frac{\partial S_{\tau}^{(\kappa)}}{\partial \kappa}=\sum_{h} \sum_{e \subset h} l_{e} \frac{\partial f_{h}^{(\kappa)}}{\partial l_{e}} \varphi_{h}^{(\kappa)}+\sum_{H} \alpha_{H}^{(\kappa)} F_{H}-\sum_{h \subset H} \sum_{e \subset h} l_{e} \frac{\partial f_{h}^{(\kappa)}}{\partial l_{e}}
$$

which, evaluated on a solution to (5.11), (5.12) results in

$$
S_{\mathcal{T}, \tau}^{(\kappa)}+\frac{2}{D-2} \kappa \frac{\partial S_{\mathcal{T}, \tau}^{(\kappa)}}{\partial \kappa}=\sum_{H} \alpha_{H}^{(\kappa)} F_{H}
$$

Note the similarity to (5.17).

The solutions for the $\alpha_{H}, \alpha_{H}^{(\kappa)}$ in fact converge to each other in the perfect limit. In order to show this, we assume that $l_{e}, \alpha_{H}$ satisfy the equations (5.4), (5.5), and $l_{e}+$ $\delta l_{e}, \alpha_{H}+\delta \alpha_{H}$ satisfy the equations (5.11), (5.12). We consider the limit of very fine triangulations $\tau$ - in particular we assume that both solutions are sufficiently close to a solution to the Einstein equations - this in particular means that the scale over which the curvature changes is much larger than $l_{e}$ or $l_{e}+\delta l_{e}$. For curved simplices the limit of small edge lengths coincides with the limit of small curvature. Expanding curved quantities in $\kappa$ results in

$$
V_{\sigma}^{(\kappa)}=V_{\sigma}+\kappa{\left.\frac{\partial V_{\sigma}^{(\kappa)}}{\partial \kappa}\right|_{\kappa=0}}+O\left(\kappa^{2}\right)
$$

In the appendix A.1 it is proved that the term linear in $\kappa$ is of order $O\left(l_{e}^{D+2}\right)$. Furthermore, for a dihedral angle $\theta_{h}^{\sigma(\kappa)}$ one has, using (A.4) and the Schlaefli identity (A.5)

$$
\sum_{h \subset \sigma} f_{h}^{(\kappa)} \theta_{h}^{\sigma(\kappa)}=\sum_{h \subset \sigma} f_{h} \theta_{h}^{\sigma}+\frac{D(D-1)}{2} \kappa V_{\sigma}+\left.\kappa \sum_{h \subset \sigma} \frac{\partial f_{h}^{(\kappa)}}{\partial \kappa}\right|_{\kappa=0} \theta_{h}^{\sigma}+O\left(\kappa^{2}\right)
$$

where quantities without superscript are volumes and angles in flat simplices. As a result, we get

$$
S_{\tau}^{(\kappa)}=S_{\tau}+\left.\kappa \sum_{h} \frac{\partial f_{h}^{(\kappa)}}{\partial \kappa}\right|_{\kappa=0} \xi_{h}+O\left(\kappa^{2}\right)
$$

where

$$
\xi_{h}:= \begin{cases}\varphi_{h}-\sum_{H \supset h} \alpha_{H} & \text { for } h \subset H \\ \varphi_{h} & \text { for } h \nsubseteq H\end{cases}
$$

Due to the Regge equations (5.4)

$$
\sum_{h \supset e} \frac{\partial f_{h}}{\partial l_{e}} \xi_{h}=\Lambda \sum_{\sigma \supset e} \frac{\partial V_{\sigma}}{\partial l_{e}}
$$


and due to the assumed regularity of the triangulation $\tau$, where the edge lengths are all of the order of magnitude of some lengths $l$, one has that $\xi_{h} \sim l^{2}$. In the limit of very fine $\tau$, both $l_{e}$ and $l_{e}+\delta l_{e}$ can expected to tend to zero, so we can expand (5.11) in $\delta l_{e}$ and compare it with $l_{e}$. We get

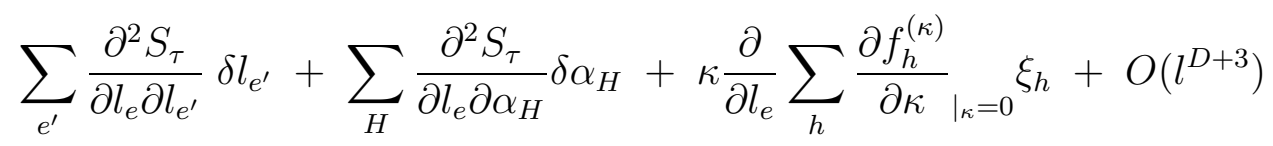

Since $\frac{\partial^{2} S_{\tau}}{\partial l_{e} \partial l_{e^{\prime}}} \sim l^{D-2}$ and $\frac{\partial^{2} S_{\tau}}{\partial l_{e} \partial \alpha_{H}} \sim l$ for $e \subset H$, we get that

$$
\begin{aligned}
\delta l_{e^{\prime}} & \sim l^{3} \\
\delta \alpha_{H} & \sim l^{D} .
\end{aligned}
$$

Hence the perfect limit $\tau \rightarrow \infty$ corresponds to the limit $l \rightarrow 0$. Therefore $\alpha_{H}^{(\kappa)}=\alpha_{H}+\delta \alpha_{H}$ converges to $\alpha_{H}$ in the continuum limit.

Furthermore, the perfect actions $S_{\mathcal{T}, *}$ and $S_{\mathcal{T}, *}^{(\kappa)}$ obviously coincide for $\kappa=\Lambda=0$. So not only do they satisfy the same ODE w.r.t. $\Lambda=(D-1)(D-2) \kappa / 2$, which is first order, they also coincide for one value. Therefore, they must coincide as functions of the $F_{H}$, and we conclude

$$
S_{\mathcal{T}, *}=\lim _{\tau \rightarrow \infty} S_{\mathcal{T}, \tau}=\lim _{\tau \rightarrow \infty} S_{\mathcal{T}, \tau}^{(\kappa)}=S_{\mathcal{T}, *}^{(\kappa)}
$$

\subsection{Constantly curved subsector}

For $D>3$ it is nontrivial to compute the perfect limit of the improved action $S_{\mathcal{T}, \tau}$ given by (5.7), since the $\alpha_{H}$ do not necessarily, unlike in $D=3$, have to have the interpretation of deficit angles at the hinges $H$ in that limit. In general, it will be quite complicated to compute the $\alpha_{H}$ in general. However, there is a special case in which one can compute the perfect action $S_{\mathcal{T}, *}$, which is when the $F_{H}$ satisfy the following requirement:

Let $\mathcal{T}$ be a triangulation of a manifold $|\mathcal{T}|=M$ with constant curvature $\kappa$ with constantly curved simplices $\Sigma$, such that there are vanishing deficit angles. If the $D-2$ dimensional hinges $H$ have a volume $F_{H}$, then the value of the perfect action $S_{\tau, *}$ on that configuration $F_{H}$ is given by

$$
S_{\mathcal{T}, *}\left(F_{H}\right)=\sum_{H \subset \partial \mathcal{T}} F_{H}\left(\pi-\sum_{\Sigma \supset H} \theta_{H}^{(\kappa) \Sigma}\right)+(D-1) \kappa V_{M}
$$

where $\theta_{H}^{(\kappa) \Sigma}$ is the dihedral angle in the curved simplex $\Sigma$ at the hinge $H$, and $V_{M}$ is the volume of the manifold $M$. This can be seen as follows: In the last section we have shown that the Regge action with curved simplices and the flat simplices lead to the same perfect action $S_{\mathcal{T}, *}$ if $\Lambda$ and $\kappa$ are related by (3.3). Therefore we can use curved simplices instead of flat ones in our triangulation $\mathcal{T}$. However, curved simplices can be glued together with vanishing deficit angles $\epsilon_{H}^{(\kappa)}=0$ to form the manifold $M$, since $M$ has constant sectional curvature $\kappa$. There are in fact infinitely many ways to do this, which can all be related by Pachner moves that do not change the boundary $\partial \mathcal{T}$. For all of 
these possibilities, the geometry satisfies trivially the Regge equations (3.10), because the deficit angles all vanish. Moreover, the constraints (5.1) are satisfied by definition. The value of the Regge action $S_{\mathcal{T}}$ does not actually depend on the exact triangulation $\mathcal{T}$, it is only depending on the boundary data, i.e. the $F_{H}$ for $H \in \partial \mathcal{T}$ and the extrinsic dihedral angles. The action (3.9) evaluated on $\epsilon_{H}^{(\kappa)}=0$ gives exactly (5.29). Since it is invariant under refinement of the triangulation, it is by definition the perfect action. Moreover, it is invariant under Pachner moves, and invariant under variations of the $F_{H}$ which result of vertex displacements, since these only change the $F_{H}$ in the triangulation, but do not change the geometry, which is that of constant curvature. Thus, in this special case we regain $D$ gauge degrees of freedom per vertex (the vertex displacements), which reflects the diffeomorphism symmetry of lapse and shift from the continuum theory.

Note that in this case (15.14) shows that $\alpha_{H} \equiv \epsilon_{H}^{(\kappa)}$ for $H \in \mathcal{T}^{\circ}$. Moreover, for the special case of $\mathcal{T}$ consisting of one simplex $\Sigma$, we can - in a similar derivation as for $D=3$, show that

$$
\sum_{H} F_{H} \frac{\partial \alpha_{H}}{\partial F_{H^{\prime}}}=(D-1) \kappa \frac{\partial V_{\Sigma}}{\partial F_{H^{\prime}}},
$$

which - since the $\frac{D(D-1)}{2} \times \frac{D(D-1)}{2}$-matrix $\partial F_{H} / \partial L_{E}$ is invertible 10 - is equivalent to the Schlaefli identity within curved simplices (A.5).

In general, the $F_{H}$ that are the arguments of the improved and the perfect action will not satisfy the requirement that there exists a triangulation of curved simplices that can be glued together with vanishing deficit angles 11 . In these cases $\alpha_{H}$ will have a much more complicated interpretation, and will be much harder to compute. In the case above where we have computed the perfect action, however, we have recovered the perfect action to reproduce a manifold with constant curvature $\kappa$, which is a solution of the continuum theory of GR, which exists in all dimensions $D$, as we have shown in chapter (3.1). For $D>3$, the sector of solutions is much larger, however, and contains many more solutions.

\section{Summary and Conclusion}

We have investigated the concept of improved and perfect actions in Regge calculus, where the reparametrization invariance of General Relativity is usually broken.

Discretizations of theories with symmetries usually lose that symmetry, e.g. in lattice gauge theory, where Poincaré-invariance is broken by introduction of a lattice. The motivation for our analysis was that the concept of improved and perfect actions is used in order to regain the symmetry within the lattice formulation. The QCD Lagrangian is not diffeomorphism invariant, however, and the techniques for lattice QCD are therefore not directly applicable to Regge Gravity.

It is well-known that one-dimensional systems with reparametrization invariance lose that symmetry upon discretization, and there is a procedure to construct improved and

\footnotetext{
${ }^{10}$ Apart from discretely many cases, see e.g. [21].

${ }^{11}$ It might not be possible to glue constantly curved simplices with these $F_{H}$ together at all - although for each separate simplex the relation between the $L_{E}$ and the $F_{H}$ can be inverted, the resulting geometries of neighboring simplices might be incompatible. One can suspect that the geometry described will not be that of constantly curved simplices, but rather of objects which are topological simplices, but have a geometry which satisfies Einstein's equations in $D$ dimensions with a cosmological constant $\Lambda$ (of which the constantly curved ones are a special case).
} 
perfect actions in this case in order to arrive at discrete actions which retain exact reparametrization invariance [6]. We have reviewed this in chapter 2, and have proposed a procedure to construct improved and perfect (classical) actions for discretized, reparametrization invariant field theories, in particular Regge Calculus in chapter 4.1. We have applied this scheme to Regge gravity in arbitrary dimensions $D$.

We have done this by considering improved actions $S_{\mathcal{T}, \tau}$ which are defined on a triangulation $\mathcal{T}$, which however incorporate the dynamics of the refined triangulation $\tau$, i.e. is closer to the actual continuum dynamics. In the canonical formulation this leads also to a better approximation of the constraints in the sense of [7]. It seems that this is a useful setting in which to think about renormalization group flow in a diffeomorphism-invariant context. Since the actual scales in the theory have to be determined dynamically, they are not available to label a cut-off for the theory, and in particular to compare them for different labels. However, one can investigate the difference of the dynamics which are discretized on two triangulations $\mathcal{T}$ and $\tau$, the continuum limit being better and better approximated the larger the difference between the two, i.e. the finer $\tau$ is compared to $\mathcal{T} 12$

We have shown that the perfect action or $3 D$ Regge calculus for $\Lambda \neq 0$ can be computed explicitly 13 It can be obtained by replacing the flat tetrahedra by tetrahedra of constant curvature $\kappa=\Lambda$. This leads to the action (4.13) which exactly reproduces the continuum dynamics of $3 D \mathrm{GR}$ with cosmological constant, i.e. vanishing deficit angles $\epsilon_{e}^{(\kappa)}=0$, leading to space-time with constant local curvature. As a consequence, the thus obtained perfect action leads to a similar vertex displacement symmetry than one finds in $3 D$ Regge calculus for $\Lambda=0$.

Since for $D>3$ the continuum theory possesses local degrees of freedom, the perfect action is much harder to construct in this case. Nevertheless, we could show that the Regge actions with flat simplices, and that with simplices of constant curvature $\kappa=\Lambda / 3$ lie in the same universality class, i.e. lead to the same perfect action $S_{\mathcal{T}, *}$. Moreover, we were able to express the perfect action in terms of the continuum limit of the Lagrange multipliers $\alpha_{H}$ and the volumes of the simplices. For the subsector of constantly curved solutions, which exists in GR for all dimensions, the $\alpha_{H}$ can in fact be computed to be the deficit(or, in case of boundary hinges $H \subset \partial \mathcal{T}$, extrinsic-) angles in constantly curved simplices, where the curvature and the cosmological constant are related by (3.3). For this subsector of solutions, the perfect action possesses the vertex displacement symmetry, which lead to $D$ gauge degrees of freedom per vertex. It therefore captures the gauge symmetry of lapse and shift, since it reproduces exactly the continuum dynamics (of constant curvature).

In this work we did not obtain explicitly an improved action which takes into account propagating degrees of freedom. This would correspond to integrating out higher frequency gravitons and their interactions and finding an effective action. We expect this to be a very complicated task leading to a non-local action. However it is a promising one with possible contacts to other quantum gravity approaches [22]. As a first step one can consider an expansion around flat space and define an action that takes into account the

\footnotetext{
${ }^{12}$ Since the triangulations $\tau$ form a partially ordered set, it might be that - in mathematical terms the renormalization group flow in this context has to be treated with the convergence of filters, rather than sequences.

${ }^{13}$ For $\Lambda=0$ the Regge action is already perfect.
} 
lowest non-linear dynamics of the gravitons [23. As the perfect action is by construction triangulation independent this could be also helpful for understanding how to obtain triangulation independent models.

The $\kappa$-curved simplices, which appear in the improved and perfect actions, can be useful for the construction of quantum gravity models for several reasons.

- Using the perfect action $S_{\mathcal{T}}^{(\kappa)}$ given by (4.13) instead of the Regge action (3.4), is a more appropriate description for the problem at hand, since for $3 D$ the perfect action correctly reflects the finite number of degrees of freedom of the continuum theory. These are not directly visible if one uses flat tetrahedra, since for $\Lambda \neq 0$ the corresponding Regge equations lead to a unique solution for the edge lengths. So no gauge freedom is apparent in this description. The edge lengths can therefore be mistaken to be physical degrees of freedom. The perfect action however is not only invariant under further refinement of the triangulation, it also shows that the edge lengths in itself are not physical, but rather are a gauge artefact introduced by a choice of triangulation.

Not only shows this that in construction of quantized models of $3 D$ Regge calculus with $\Lambda \neq 0$ the perfect action $S_{\mathcal{T}}^{(\kappa)}$ might be more suitable than $S_{\mathcal{T}}$, in a broader context it shows how in discretized gravity theories it can be difficult to tell physical from gauge degrees of freedom.

This is in particular important in $4 D$, where the solutions to the Regge equations (even for $\Lambda=0$ ) are generically unique. This is usually taken as proof that the diffeomorphism symmetry of GR has been successfully divided out, and one is only working with gauge-invariant quantities (i.e. the edge lengths), since the gauge symmetry of GR, apparent in the non-uniqueness of solutions to the boundary value problem, vanishes in the discrete theory. However, in the light of the analysis of [7] and this article, one might consider that not all of the configuration variables of Regge calculus might be in fact physical. Rather, by constructing a perfect action for discretized gravity, which reflects the continuum dynamics and hence the gauge symmetries of GR, one might get more insight into which of the degrees of freedom are actually physical, and which are gauge. This is in particular important in attempts to quantize discrete gravity theories by using Regge triangulations, as happens in Spin Foams.

We therefore suggest that it might be valuable to study how gauge degrees of freedom are regained in the continuum limit, and think that the improved and perfect actions presented in this article can be helpful in this pursuit.

- In particular the usage of simplices with constant curvature might be useful for first order formulations in Regge calculus, and the questions of constraints in this context [5, 7, 8. Furthermore, an area-angle formulation [24 with simplices of constant (nonzero) curvature might be more viable than in the flat case, since e.g. in $4 D$ the 10 dihedral angles of a 4-simplex determine its geometry completely, not just its conformal structure as with flat simplices. These variables are not only appropriate for spin foam models but seem also to be useful to obtain a canonical formulation [25]. See [26] for formulations based on different sets of basic variables 
and a first order formulation involving $\kappa$-curved simplices. Curved simplices have been proposed in [27], but no action has been proposed there. In general quantum gravity models with a positive cosmological constant are better behaved in the infrared and can even serve as regulators for models without a cosmological constant. Therefore it seems useful to investigate the construction of spin foam models with a cosmological constant.

- The Turaev-Viro invariant [28] for 3-manifolds reproduces in the semiclassical limit the geometry of constantly curved simplices for $\Lambda>0$ [29]. The construction of corresponding Spin Foam models for $\Lambda<0$, which is still elusive, could benefit form the formalism presented here by starting a quantization of the perfect action (4.13) for $\Lambda<0$. In general we note that for the $3 D, \Lambda>0$ case a quantization having the perfect action as a limit is available (namely the Turaev-Viro models), whereas a similar quantization based on the non-perfect action is missing. In the canonical formulation one has to worry about complicated factor ordering ambiguities [30] in addition to an anomalous constraint algebra. In contrast a quantization based on $\kappa$-curved simplices could avoid these issues.

In general it would be interesting to see whether a similar procedure for reobtaining gauge symmetries (and triangulation independence) as presented here for the classical theory works also for the quantum theory. The Ponzano-Regge with an added cosmological term and the Tuarev-Viro model would be an interesting example [31. See also [32] where spatial diffeomorphism symmetry has been reobtained in the continuum limit for a symmetry reduced model.

\section{Acknowledgements}

The authors would like to thank John Barrett, Simone Speziale, Ruth Williams and Jose Zapata for valuable discussions and remarks. Funding of B.B. by ESF grant PESC/2805 within the Quantum Geometry and Quantum Gravity network for a visit in Utrecht is gratefully acknowledged. B.B. would like to thank for the hospitality at the ITF, Utrecht. The research of B.D. at the University of Utrecht was supported by a Marie-Curie fellowship of the European Union.

\section{A Curved Simplices}

In the following, let $\sigma$ denote a $D$-dimensional simplex of constant curvature $\kappa$. Denote its $D$-dimensional volume by $V_{\sigma}^{(\kappa)}$. A hinge $h$ is a $D-2$ dimensional subsimplex (which is again a simplex of constant curvature $\kappa$ ), and we denote its $D$-2-dimensional volume by $F_{h}^{(\kappa)}$. For a hinge $h \subset \sigma$ denote the interior deficit angle between the two $D$-1-dimensional subsimplices of $\sigma$ meeting at $h$ by $\theta_{h}^{(\kappa)}$.

The simplex $\sigma$ is completely determined by the lengths of its $N:=\frac{D(D+1)}{2}$ edges (the 1-simplices). All of the above are regarded as functions of their lengths $L_{1}, \ldots, L_{N}$.

If we numerate the vertices of $\sigma$ from 1 to $D+1$, we specify a subsimplex by $(i j \ldots k)$ if it is spanned by the vertices with the numbers $i, j \ldots, k$, and by $[i j \ldots k]$ if it is spanned 
by all vertices except $i, j, \ldots, k$. In this notation an edge can be denoted as $e=(i j)$, and its dual hinge by $h=[i j]$.

Denote the geodesic lengths of the edges $(i j)$ by $L_{(i j)}$. Then the $(D+1) \times(D+1)$ matrix $G$ with entries

$$
G_{i j}=c_{\kappa}\left(L_{(i j)}\right)
$$

where the function $c_{\kappa}(x)$ is defined by

$$
c_{\kappa}(x):= \begin{cases}\cos (\sqrt{\kappa} x) & \kappa>0 \\ \cosh (\sqrt{-\kappa} x) & \kappa<0\end{cases}
$$

is called the Gram matrix of the simplex. We denote by $G^{i j}$ the inverse of $G_{i k}$. Then the interior dihedral angle $\theta_{[i j]}$ opposite of the edge $(i j)$ is given by 33

$$
\cos \theta_{[i j]}^{(\kappa)}=-\frac{G^{i j}}{\sqrt{G^{i i}} \sqrt{G^{j j}}} .
$$

Hence, for any hinge $h$ the exterior angle $\theta_{h}^{(\kappa)}$, regarded as a function of the lengths $L_{1}, \ldots, L_{N}$, exhibits the scaling behavior 14

$$
\theta_{h}^{(\kappa)}\left(L_{1}, \ldots, L_{N}\right)=\theta_{h}^{(1)}\left(\sqrt{\kappa} L_{1}, \ldots, \sqrt{\kappa} L_{N}\right) .
$$

As a result we have

$$
\frac{\partial}{\partial \kappa} \theta_{h}^{(\kappa)}=\frac{1}{2 \kappa} \sum_{e \subset \sigma} L_{e} \frac{\partial \theta_{h}^{(\kappa)}}{\partial L_{e}}
$$

Furthermore, the geometric quantities in curved simplices satisfy the Schläfli identity

$$
\sum_{h \subset \sigma} F_{h}^{(\kappa)} \frac{\partial \theta_{h}^{\sigma(\kappa)}}{\partial L_{e}}=(D-1) \kappa \frac{\partial V_{\sigma}^{(\kappa)}}{\partial L_{e}} \quad \text { for all edges } e \subset \sigma
$$

\section{A.1 Geometric Identities in curved simplices}

In this section we derive a generalization of Euler's theorem

$$
\frac{1}{D} \sum_{e \subset \sigma} L_{e} \frac{\partial V_{\sigma}}{\partial L_{e}}=V_{\sigma}
$$

to curved tetrahedra.

Lemma A.1. For a simplex $\sigma$ of dimension $D$ and constant curvature we have

$$
V_{\sigma}^{(\kappa)}\left(s L_{1}, \ldots, s L_{N}\right)=s^{D} V_{\sigma}^{\left(\kappa s^{2}\right)}\left(L_{1}, \ldots, L_{N}\right)
$$

Proof: This can in fact be seen easily for $\kappa>0$, where the simplex is a subset of a $D$-dimensional sphere of radius $R=1 / \sqrt{\kappa}$. If the radius is scaled by $s$, as well as all the edge lengths, the volume of the sphere is scaled by $s^{D}$, hence also the volume of the simplex. For $\kappa<0$ a similar reasoning for hyperbolic spheres applies. The formula (A.7) follows.

\footnotetext{
${ }^{14}$ The formulae presented here hold for $\kappa>0$. For $\kappa<0$ analogous formulae can be deduced.
} 
Corollary A.1. For any D-dimensional simplex $\sigma$ of constant curvature $\kappa$ we have

$$
\frac{\partial}{\partial s} V_{\sigma}^{(\kappa)}\left(L_{1}, \ldots, L_{N}\right)=s^{D-1} \sum_{e \subset \sigma} L_{e} \frac{\partial}{\partial L_{e}} V_{\sigma}^{\left(\kappa s^{2}\right)}\left(L_{1}, \ldots, L_{N}\right) .
$$

Proof: By explicit calculation:

$$
\begin{aligned}
\frac{\partial}{\partial s} V_{\sigma}^{(\kappa)}\left(s L_{1}, \ldots, s L_{N}\right) & =\sum_{e \subset \sigma} L_{e} \frac{\partial}{\partial\left(s L_{e}\right)} V_{\sigma}^{(\kappa)}\left(s L_{1}, \ldots, s L_{N}\right) \\
& =\frac{1}{s} \sum_{e \subset \sigma} L_{e} \frac{\partial}{\partial L_{e}} V_{\sigma}^{(\kappa)}\left(s L_{1}, \ldots, s L_{N}\right) \\
& =s^{D-1} \sum_{e \subset \sigma} L_{e} \frac{\partial}{\partial L_{e}} V_{\sigma}^{\left(\kappa s^{2}\right)}\left(L_{1}, \ldots, L_{N}\right) .
\end{aligned}
$$

This was the claim.

Another important identity is the following generalization of Euler's formula to curved simplices:

Lemma A.2. If $\sigma$ is a $D$-dimensional simplex of constant curvature $\kappa$, then

$$
\frac{1}{D} \sum_{e \subset \sigma} L_{e} \frac{\partial V_{\sigma}^{(\kappa)}}{\partial L_{e}}=V_{\sigma}^{(\kappa)}+\frac{2}{D} \kappa \frac{\partial V_{\sigma}^{(\kappa)}}{\partial \kappa}
$$

Proof: We prove this by induction over $D$, and first note that it is trivially true for $D=1$. The case $D=2$ can be shown explicitly by recalling the formula for the area of a spherical (or hyperbolical) triangle $t$

$$
V_{t}^{(\kappa)}=\frac{\theta_{1}^{(\kappa)}+\theta_{2}^{(\kappa)}+\theta_{3}^{(\kappa)}-\pi}{\kappa}
$$

where the $\theta_{i}^{(\kappa)}$ are the interior angles of $t$. Since they are interior dihedral angles of curved simplices, they satisfy the relations (A.4). This leads to

$$
\frac{\partial V_{t}^{(\kappa)}}{\partial \kappa}=-\frac{V_{t}^{(\kappa)}}{\kappa}+\frac{1}{2 \kappa} \sum_{i=1}^{3} L_{i} \frac{\partial V_{t}^{(\kappa)}}{\partial L_{i}} .
$$

This shows (A.9) for $D=2$. We now show that the formula is true for $D$ if it is true for $D-2$. We begin with Schlaefli's formula 34]

$$
(D-1) \kappa d V_{\sigma}^{(\kappa)}=\sum_{h \subset \sigma} F_{h}^{(\kappa)} d \theta_{h}^{(\kappa)}
$$

As a consequence, we have (whenever a function appears without arguments, it is supposed to be taken at the point $\left.\left(L_{1}, \ldots, L_{N}\right)\right)$ :

$$
\begin{aligned}
(D-1) \kappa V_{\sigma}^{(\kappa)} & =\int_{0}^{1} d s \sum_{h \subset \sigma} F_{h}^{(\kappa)}\left(s L_{1}, \ldots, s L_{n}\right) \frac{\partial}{\partial s} \theta_{h}^{(\kappa)}\left(s L_{1}, \ldots, s L_{N}\right) \\
& =\sum_{h \subset \sigma} F_{h}^{(\kappa)} \theta_{h}^{(\kappa)}-\int_{0}^{1} d s \sum_{h \subset \sigma} \theta^{\left(\kappa s^{2}\right)}\left(L_{1}, \ldots, L_{N}\right) \frac{\partial}{\partial s} F_{h}^{(\kappa)}\left(s L_{1}, \ldots s L_{N}\right)
\end{aligned}
$$


Remembering that each hinge $h$ is a $D-2$ dimensional simplex of constant curvature $\kappa$, we conclude with (A.8) that

$$
\begin{aligned}
S^{(\kappa)}:=-\sum_{h \subset \sigma} F_{h}^{(\kappa)} \theta_{h}^{(\kappa)}+(D-1) \kappa V_{\sigma}^{(\kappa)} & =-\int_{0}^{1} d s s^{D-3} \sum_{h \subset \sigma} \theta_{h}^{\left(\kappa s^{2}\right)} \sum_{e \subset h} L_{e} \frac{\partial F_{h}^{\left(\kappa s^{2}\right)}}{\partial L_{e}} \\
& =-\frac{1}{2} \kappa^{-\frac{D-2}{2}} \int_{0}^{\kappa} d y y^{\frac{D-4}{2}} \sum_{h \subset \sigma} \theta_{h}^{(y)} \sum_{e \subset h} L_{e} \frac{\partial F_{h}^{(y)}}{\partial L_{e}}
\end{aligned}
$$

where we have used a change of variable $y=\kappa s^{2}$.

We now derive the two different ways (A.13) of writing $S^{(\kappa)}$ with respect to $\kappa$. The first one gives us

$$
\begin{aligned}
\frac{\partial S^{(\kappa)}}{\partial \kappa} & =\frac{\partial}{\partial \kappa}\left(-\sum_{h \subset \sigma} F_{h}^{(\kappa)} \theta_{h}^{(\kappa)}+(D-1) \kappa V_{\sigma}^{(\kappa)}\right) \\
& =-\sum_{h \subset \sigma} \frac{F_{h}^{(\kappa)}}{\partial \kappa} \theta_{h}^{(\kappa)}-\sum_{h \subset \sigma} F_{h}^{(\kappa)} \frac{\partial \theta_{h}^{(\kappa)}}{\partial \kappa}+(D-1) V_{\sigma}^{(\kappa)}+(D-1) \kappa \frac{\partial V_{\sigma}^{(\kappa)}}{\partial \kappa}(A)
\end{aligned}
$$

Note that with (A.4) and (A.12) we have

$$
\begin{aligned}
\sum_{h \subset \sigma} F_{h}^{(\kappa)} \frac{\partial \theta_{h}^{(\kappa)}}{\partial \kappa} & =\frac{1}{2 \kappa} \sum_{h \subset \sigma} F_{h}^{(\kappa)} \sum_{e \subset \sigma} L_{e} \frac{\partial V_{\sigma}^{(\kappa)}}{\partial L_{e}} \\
& =\frac{D-1}{2} \sum_{e \subset \sigma} L_{e} \frac{\partial V_{\sigma}^{(\kappa)}}{\partial L_{e}} .
\end{aligned}
$$

Now we use the induction hypothesis, which means that (A.9) in particular holds for $h$, i.e.

$$
\frac{\partial F_{h}^{(\kappa)}}{\partial \kappa}=\frac{1}{2 \kappa} \sum_{e \subset h} L_{e} \frac{\partial F_{h}^{(\kappa)}}{\partial L_{e}}-\frac{D-2}{2 \kappa} F_{h}^{(\kappa)} .
$$

Inserting (A.15) and (A.16) into (A.14) we arrive at

$$
\begin{aligned}
\frac{\partial S^{(\kappa)}}{\partial \kappa}= & -\frac{1}{2 \kappa} \sum_{h \subset \sigma} \theta_{h}^{(\kappa)} \sum_{e \subset h} L_{e} \frac{\partial F_{h}^{(\kappa)}}{\partial L_{e}}+\frac{D-2}{2 \kappa} \sum_{h \subset \sigma} \theta_{h}^{(\kappa)} F_{h}^{(\kappa)} \\
& -\frac{D-1}{2} \sum_{e \subset \sigma} L_{e} \frac{\partial V_{\sigma}^{(\kappa)}}{\partial L_{e}}+(D-1) V_{\sigma}^{(\kappa)}+(D-1) \kappa \frac{\partial V_{\sigma}^{(\kappa)}}{\partial \kappa} .
\end{aligned}
$$

On the other hand, by (A.13) we have 


$$
\begin{aligned}
\frac{\partial S^{(\kappa)}}{\partial \kappa} & =\frac{\partial}{\partial \kappa}\left(-\frac{1}{2} \kappa^{-\frac{D-2}{2}} \int_{0}^{\kappa} d y y^{\frac{D-4}{2}} \sum_{h \subset \sigma} \theta_{h}^{(y)} \sum_{e \subset h} L_{e} \frac{\partial F_{h}^{(y)}}{\partial L_{e}}\right) \\
& =-\frac{D-2}{2 \kappa} S^{(\kappa)}-\frac{1}{2 \kappa} \sum_{h \subset \sigma} \theta_{h}^{(\kappa)} \sum_{e \subset h} L_{e} \frac{\partial F_{h}^{(\kappa)}}{\partial L_{e}} \\
& =\frac{D-2}{2 \kappa} \sum_{h \subset \sigma} \theta_{h}^{(\kappa)} F_{h}^{(\kappa)}-\frac{(D-1)(D-2)}{2} V_{\sigma}^{\kappa}-\frac{1}{2 \kappa} \sum_{h \subset \sigma} \theta_{h}^{(\kappa)} \sum_{e \subset h} L_{e} \frac{\partial F_{h}^{(\kappa)}}{\partial L_{e}} .
\end{aligned}
$$

By comparing (A.17) and (A.18) we arrive at

$$
\left.-\frac{D-1}{2} \sum_{e \subset \sigma} L_{e} \frac{\partial V_{\sigma}^{(\kappa)}}{\partial L_{e}}+(D-1) V_{\sigma}^{(\kappa)}+(D-1) \kappa \frac{\partial V_{\sigma}^{(\kappa)}}{\partial \kappa}=-\frac{(D-1)(D-2)}{2} V_{\sigma}^{(\eta)} \mathrm{A} .19\right)
$$

which is equivalent to (A.9).

There is an important corollary: Deriving (A.9) w.r.t $\kappa$ and setting $\kappa=0$, one can see that

$$
\left.(D+2) \frac{\partial V_{\sigma}^{(\kappa)}}{\partial \kappa}\right|_{\kappa=0}=\left.\sum_{e \subset \sigma} l_{e} \frac{\partial}{\partial l_{e}} \frac{\partial V_{\sigma}^{(\kappa)}}{\partial \kappa}\right|_{\kappa=0}
$$

which, by Euler's theorem, shows that $\left.\frac{\partial V_{\sigma}^{(\kappa)}}{\partial \kappa}\right|_{\kappa=0}$ is a homogenous function of the edge lengths $l_{e}$ of degree $D+2$. An explicit example for this is e.g. $D=2$, where one can, with (A.10), show that

$$
\left.\frac{\partial a_{t}^{(\kappa)}}{\partial \kappa}\right|_{\kappa=0}=\frac{1}{24} \sum_{e \subset t} l_{e}^{2} a_{t}^{(\kappa=0)}
$$

which is indeed homogenous of degree 4 .

\section{References}

[1] Wald R.: General Relativity 1884 Chicago University Press

[2] Regge T.: General relativity without coordinates (1961) Nuovo Cim. 19 558-571

[3] Roček M., Williams R.: Quantum Regge calculus (1981) Phys. Lett. B 1 31-37 Williams R.: Recent progress in Regge Calculus (1997) Nucl. Phys. Proc. 57 73-81 arXiv:gr-qc/9702006

[4] Baez J.: Spin Foam Models (1998) Class. Quant. Grav. 151827 arXiv:gr-qc/9709052, Freidel L., Krasnov K.: Spin Foam Models and the Classical Action Principle (1999) Adv. Theor. Math. Phys. 2 1183, Oriti, D.: Spacetime geometry from algebra: spin foam models for non-perturbative quantum gravity (2001) Rept. Prog. Phys. 64 1489; Perez A.: Spin Foam Models for Quantum Gravity (2003) Class. Quant. Grav. 20 R43 arXiv:gr-qc/0301113 
[5] Dittrich B.: Diffeomorphism symmetry in quantum gravity models (2009) Adv. Sci. Lett. 2 121-132 [arXiv:0810.3594 [gr-qc]]

[6] Marsden J., West M.: Discrete mechanics and variational integrators Acta Numerica 10 (2001) 357

[7] Bahr B., Dittrich B.: (Broken) Gauge Symmetries and Constraints in Regge Calculus arXiv:0905.1670

[8] Gambini R., Pullin J.: Consistent discretization and canonical classical and quantum Regge calculus (2006) Int.J.Mod.Phys. D 15 1699-1706 arXiv:gr-qc/0511096], Gambini R., Pullin J.: Consistent discretization as a road to quantum gravity arXiv:gr-qc/0512065 Campiglia M., Di Bartolo C., Gambini R., Pullin J.: Uniform discretizations: a new approach for the quantization of totally constrained systems (2006) Phys.Rev.D 74124012 arXiv:gr-qc/0610023, Gambini R., Lewandowski J., Marolf D., Pullin J.:On the consistency of the constraint algebra in spin network quantum gravity (1998) Int.J.Mod.Phys.D 7 97-109 arXiv:gr-qc/9710018

[9] Thiemann T. The Phoenix Project: Master Constraint Programme for Loop Quantum Gravity 2006 Class. Quant. Grav. 23, 2211 arXiv:gr-qc/0305080, Thiemann T. Quantum Spin Dynamics VIII. The Master Constraint 2006 Class. Quant. Grav. 23, 2249 arXiv:gr-qc/0510011 Dittrich B., Thiemann T.: Testing the Master Constraint Programme for Loop Quantum Gravity I. General Framework (2006) Class.Quant.Grav. 23 1025-1066 arXiv:gr-qc/0411138

[10] Gupta R.: Introduction to Lattice QCD arXiv:hep-lat/9807028

[11] Hasenfratz P., Niedermayer F.: Perfect lattice actions for asymptotically free theories (1994) Nucl. Phys. B 414 785-814 [arXiv:hep-lat/9308004

[12] Wilson K., Kogut J.: The renormalization group and the epsilon expansion (1974) Phys. Rept. 12 75-200

[13] Bietenholz W., Wiese U.: Perfect Lattice action for quarks and gluons (1996) Nucl. Phys. B 464 319-352 arXiv:hep-lat/9510026], Bietenholz W., Brower R., Chandrasekharan S., Wiese U.: Progress on perfect lattice action for QCD (1997) Nucl. Phys. Proc. Suppl. 53 921-934 arXiv:hep-lat/9608068

[14] Miller W. A.: The geometrodynamic content of the Regge equations as illuminated by the boundary of a boundary principle (1986) Found. Phys. 16 143. Hamber H., Kagel G.: Exact Bianchi identities in Regge Gravity (2004) Class. Quant. Grav 21 5915-5948 arXiv:gr-qc/0107031 Gentle A. P., Kheyfets A., McDonald J. R., Miller W. A.: A Kirchoff-like conservation law in Regge calculus (2009) Class. Quant. Grav. 26015005 arXiv:0807.3041 [gr-qc]].

[15] Freidel L., Louapre D.: Diffeomorphisms and spin foam models (2003) Nucl. Phys. B 662279 arXiv:gr-qc/0212001.

[16] Piran T., Strominger A.: Solutions to the Regge equations (1986) Class. Quant. Grav. 3 97-102 
[17] Rovelli C.: Partial observables 2002 Phys.Rev. D 65124013 [arXiv:gr-qc/0110035], Dittrich B.: Partial and complete observables for Hamiltonian constrained systems (2007) Gen. Rel. Grav. 391891 arXiv:gr-qc/0411013. Partial and Complete Observables for Canonical General Relativity (2006) Class. Quant. Grav. 236155 arXiv:gr-qc/0507106.

[18] Rovelli C.: Quantum Gravity (Cambridge Monographs on Mathematical Physics) Cambridge University Press 2004

[19] Thiemann T.: Modern Canonical Quantum General Relativity (Cambridge Monographs on Mathematical Physics) Cambridge University Press 2007

[20] Hartle J., Sorkin R.: Boundary Terms In The Action For The Regge Calculus (1981) Gen.Rel.Grav. 13 541-549

[21] Barrett J. W.: First order Regge calculus (1994) Class. Quant. Grav. 112723 arXiv:hep-th/9404124.

[22] Reuter M., Weyer H.: Functional Renormalization Group Equations, Asymptotic Safety, and Quantum Einstein Gravity (2008) Lecture notes MZ-TH-07-05 arXiv:0708.1317 [hep-th]]

[23] Bahr B., Dittrich B., Höhn P.: to appear

[24] Dittrich B., Speziale S.: Area-angle variables for general relativity (2008) New J.Phys. 10083006 arXiv:0802.0864 [gr-qc]]

[25] Dittrich B., Ryan J. P.: Phase space descriptions for simplicial 4d geometries arXiv:0807.2806 [gr-qc].

[26] Bahr B., Dittrich B.: Regge calculus from a new angle [arXiv:0907.4325 [gr-qc]]

[27] Dittrich B., Freidel L., Speziale S.: Linearized dynamics from the 4-simplex Regge action (2007) Phys. Rev. D 76104020 [arXiv:0707.4513 [gr-qc]].

[28] Turaev V. G., Viro O. Y.: State sum invariants of 3 manifolds and quantum 6j symbols (1992) Topology 31 865-902

[29] Taylor Y. U., Woodward C. T.: Spherical Tetrahedra and Invariants of 3-manifolds arXiv:math/0406228

[30] Perez A.: private communication

[31] Bahr B., Dittrich B.: work in progress

[32] Gambini R., Pullin J.: Emergent diffeomorphism invariance in a discrete loop quantum gravity model arXiv:0807.2808 [gr-qc].

[33] Kokkendorff S. L.: Gram Matrix Analysis of Finite Distance Spaces in Constant Curvature (2004) Discrete Comp. Geom. 31 515-543

[34] Rivin I., Schlenker J.-M.: On the Schlaefli differential formula arXiv:math/0001176 